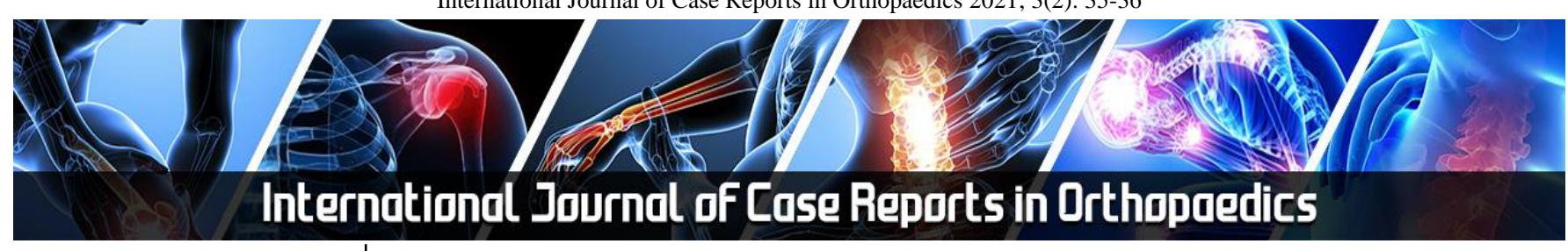

E-ISSN: 2707-8353 P-ISSN: 2707-8345 IJCRO 2021; 3(2): 35-36 Received: 19-05-2021 Accepted: 21-06-2021

Rahul Ramanathan BS (1) Carle Illinois College of Medicine, $807 \mathrm{~S}$ Wright St, Champaign, IL 61820, United States

(2) Carle Foundation Hospital, United States

Robert Bane MD

Carle Foundation Hospital, United States
Corresponding Author: Rahul Ramanathan BS (1) Carle Illinois College of Medicine, $807 \mathrm{~S}$ Wright St, Champaign, IL 61820 , United States

(2) Carle Foundation Hospital, United States

\section{Latissimus repair in wakeboarding incident: An operative case report}

\author{
Rahul Ramanathan BS and Robert Bane MD
}

DOI: $\underline{\text { https://doi.org/10.22271/27078345.2021.v3.i2a.64 }}$

\begin{abstract}
Latissimus dorsi (LD) tears are clinically uncommon and most often occur in professional baseball pitchers. Current literature does not offer a consensus for operative vs. conservative management of LD tears. The case described herein involves a wakeboarding-related LD avulsion with a discussion of factors leading to the clinical decision of operative management. A single incision, curvilinear axillary approach is outlined with intensive postoperative physical rehabilitation and complete recovery achieved within 12 months. Multiple different operative techniques have been described, including a dual-incision technique with a deltopectoral approach as well as the single-incision curvilinear posterior axillary approach described in this report. However, there are sparse comparative studies between these operative approaches. This report therefore recounts a successful case of managing LD injury operatively and in support of the single incision approach.
\end{abstract}

Keywords: latissimus dorsi, wakeboarding, case report

\section{Introduction}

Proximal upper extremity sports injuries are common in elite athletes, and the current literature focuses on common injuries, such as ulnar collateral ligament tears, labrum injuries, and rotator cuff tears. However, reports on tears of the latissimus dorsi (LD) remain sparse both clinically and in the literature ${ }^{[1]}$. Latissimus dorsi (LD) tears are clinically rare and typically present in high-performance athletes with eccentric tendon overload. Baseball pitchers are the primary high-risk population for such an injury, although there have been a few cases reported in Cross Fit, golf, and tennis athletes. Despite multiple accounts of both operative and conservative management, consensus on specific indications for each has not been thoroughly established. Additionally, multiple operative approaches have been described with sparse comparative literature ${ }^{[1]}$.

\section{Case description}

Patient was an athletic 27 -year-old male presenting with 6/10 sharp right shoulder pain. Upon further questioning, he stated that he had experienced a "pop" in his right posterior shoulder when being pulled by a boat during wakeboarding. Physical examination revealed a lump with marked localized swelling under the right axilla. Shoulder ROM tests revealed aggravated pain during abduction/extension of the arm and extension of the elbow. Full chest MRI demonstrated a full-thickness avulsion of the LD tendon from the humeral insertion point with mild retraction and no other joint complex injuries. Due to the extent of injury and the patient's interest in continuing to weightlift and participate in high-performance activities, an operative approach was heavily considered and the patient was debriefed on the procedure, risks, and prognosis. A single-incision curvilinear axillary approach was chosen to minimize the number of incisions and to aid in healing. Operative protocol involved careful dissection of the axilla, with identification and avoidance of critical structures such as the posterior brachial cutaneous nerve, radial nerve, and axillary nerve. Moderate retraction of the LD warranted deep dissection and retrieval of the tendon. Operative intervention was scheduled within 2 weeks of injury onset, allowing easy mobilization of the LD tendon. The stump of the LD was secured with three \#2 Fiber Wire sutures placed in Krackow fashion. These were then passed through MiTek G2 titanium anchors for attachment to the intertubercular groove of the humerus. Patient's arm was internally rotated prior to anchor placement to maximize visualization of the attachment site. Both the wound and prior hematoma were irrigated and the patient was then closed and placed in a shoulder immobilizer. 
Post-operatively, a stringent physical therapy program was started, with strengthening exercises beginning at 6 weeks post-op. At a 3-month follow-up, the patient had begun gentle weight training and had mildly limited internal rotation compared to his healthy arm. At 12 months followup, the patient had a DASH score of 4 out of 100 and an ASES score of 100 out of 100 , indicating that he no longer considers his upper limb disorder a problem. Additionally, the patient stated that he is back to baseline strength and has no pain or soreness during any motion other than wide-grip pull-ups, which directly engage the attachment point of the latissimus dorsi ${ }^{[2]}$.

\section{Case Discussion}

Clinical decisions regarding the management of LD tears have been varied in the literature and do not seem to offer a consensus. Previous groups have reported favorable outcomes in the management of LD tears in baseball pitchers both operatively and non-operatively ${ }^{[3]}$. Clinical decision-making in these cases may be largely dependent on the preferred lifestyle of the patient and the degree of injury. In this particular case, the patient's desire to continue weightlifting and bodybuilding was a prominent factor in the choice of management. Additionally, the latissimus in this patient was deemed to be a large and powerful muscle that could return to preoperative strength and cosmetic appearance with acute repair. Operatively, multiple approaches have been previously described, including a dual-incision technique with a deltopectoral approach and a single-incision curvilinear posterior axillary approach ${ }^{[3]}$. Comparative studies of different surgical techniques have not yet been conducted and thus the choice of technique is currently at the discretion of the surgeon. The singleincision curvilinear method chosen for this particular patient provides the benefit of using a single incision for both tendon retrieval and footprint exposure. Drawbacks to this approach include requiring an extensive knowledge of axillary neuroanatomy due to the risk of damage to critical limb structures. This case report provides support for the curvilinear posterior axillary approach to LD repair by demonstrating an excellent postoperative course and rehabilitation to baseline levels at 12 months.

\section{Imaging}

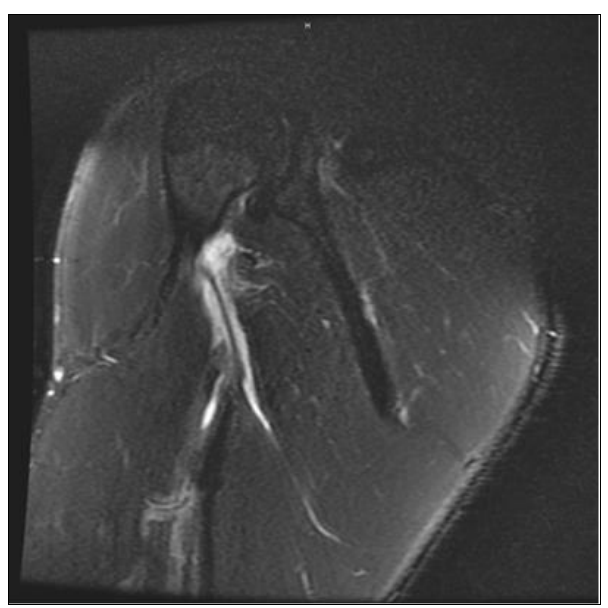

Fig 1: Preoperative coronal slice T1-weighted MRI showing the patient's right shoulder with full-thickness avulsion of the LD tendon with surrounding edema.

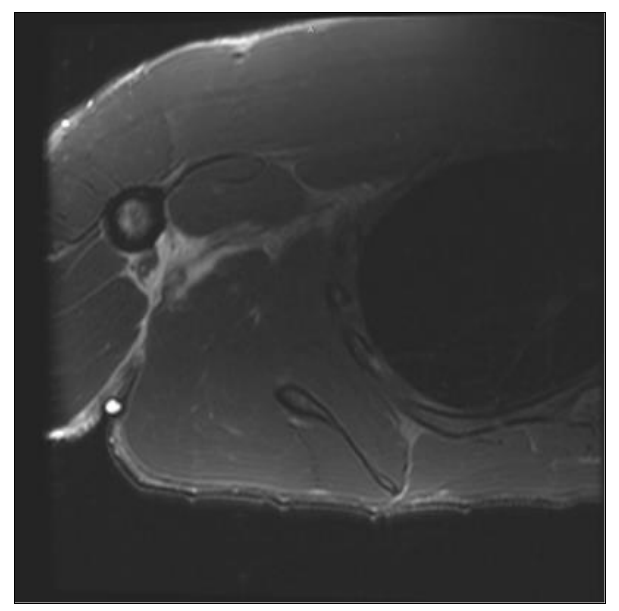

Fig 2: Preoperative axial T1-weighted view of the patient's right shoulder showing full-thickness avulsion of LD tendon with mild retraction and surrounding edema.

\section{Data Availability}

No data were used to support this study. The authors may be contacted for a report of operative techniques and postoperative rehabilitation schedule.

\section{Conflicts of Interest}

None to declare.

\section{Funding}

None received.

\section{References}

1. Alrabaa Rami George, Christopher Ahmad S Latissimus Dorsi Tendon Repair. Arthroscopy Techniques, Arthroscopy Association of North America 2020;9(2):267-73. Doi:10.1016/j.eats.2019.10.006.

2. Williams Nerys. DASH. Occupational Medicine, Oxford Academic 2014;64(1):67-68.

Doi: $10.1093 /$ occmed/kqt130.

3. Erickson Brandon $\mathrm{J}$ et al. Approach to Latissimus Dorsi and Teres Minor Injuries in the Baseball Pitcher. Current Reviews in Musculoskeletal Medicine, Current Reviews in Musculoskeletal Medicine 2019;12(1):2429. Doi: 10.1007/s12178-019-09532-y.

4. Friedman Michael V et al. Traumatic Tear of the Latissimus Dorsi Myotendinous Junction: Case Report of a CrossFit-Related Injury. Sports Health 2015;7(6):548-52. Doi: 10.1177/1941738115595975.

5. Donohue Benjamin F et al. Sports Injuries to the Latissimus Dorsi and Teres Major. American Journal of Sports Medicine 2017;45(10):2428-35. Doi: 10.1177/0363546516676062. 